\title{
The Corrosion of Secondary Phase Precipitates in Zircaloy in Superheated Water
}

\author{
Ian MacLaren ${ }^{1}$, Kirsty J. Annand ${ }^{1}$ and Mhairi Gass ${ }^{2}$ \\ 1. School of Physics and Astronomy, University of Glasgow, Glasgow \\ 2. AMEC Foster Wheeler, Clean Energy, Walton House, Birchwood Park, UK
}

It is well known that alloying zirconium with other elements is essential to achieving controllable and predictable corrosion behavior in contact with the high temperature water that is used as a reactor coolant. Such alloying results in the formation of secondary phase precipitates (SPPs), such as chromium and iron rich precipitates in Zircaloy 4. What is less well understood is the role that precipitates play in the corrosion process. There have been a number of studies of such precipitates, mainly focused on Laves phase precipitates (nominally $\mathrm{Zr}(\mathrm{Cr}, \mathrm{Fe})_{2}$ ) [1-6]. Nevertheless, such studies have mainly concentrated on imaging, point chemical analysis with EDX spectroscopy, or electron diffraction without combining all three techniques. The current paper will demonstrate that a combination of imaging, chemical mapping and diffraction mapping is necessary to understand the oxidation of such precipitates in detail.

Laves phase $\mathrm{Zr}(\mathrm{Cr}, \mathrm{Fe})_{2}$ precipitates are frequently found in Zircaloy 4 and the elemental quantification of such precipitates in the unoxidized metal show compositions consistent with expectations. One slight surprise is found, however, in that the surface of such precipitates is enriched in oxygen. On oxidation by exposure to superheated water at $360^{\circ} \mathrm{C}$, however, such precipitates undergo a complex set of changes and typically end up as veined structures as shown in Figure 1. The body of the precipitate ends up as a chromium-zirconium rich oxide with a very fine nanocrystalline (almost amorphous) structure with a well defined ring at an angle corresponding to about $2.7 \AA$. This spacing could correspond to $\mathrm{Cr}_{2} \mathrm{O}_{3}$ or to some spacings in a stabilized $\mathrm{ZrO}_{2}$, but it is unclear which is the case, or whether it is a nanoscale mixture of both. Meanwhile, Fe-rich veins are formed, which contain rather little oxygen. Moreover, these turn out to be single crystal bcc ferrite, as shown in the diffraction pattern. This suggests that the chromium and zirconium oxidize to a mixed oxide that does not have a high iron solubility, pushing the iron ahead of the oxidation front until it enriches enough to form a vein of iron, and that this process repeats several times in oxidizing the SPP.

In addition to this, other secondary phase precipitates are occasionally found in the metal with a composition close to $\mathrm{Zr}_{2} \mathrm{Fe}$. These oxidize in a totally different way to the Laves phase precipitates. Instead of forming a banded structure, a nanocrystalline structure is found, which appears to be chemically homogeneous down to the nanoscale. This is shown in Figure 2. There is a clear crystal structure to such particles, and they display a strong crystallographic texture, despite clearly being composed of many nanocrystals. Unfortunately determining the crystal structure unambiguously has proved difficult since the diffraction pattern would suggest a cubic structure with a lattice parameter around $4.7 \AA$, which does not easily fit to well-known $\mathrm{Zr}$ or Fe oxides.

An interesting fact that is observed is that the oxidised $\mathrm{Zr}_{2} \mathrm{Fe}$ SPPs usually have lateral cracks adjacent in the $\mathrm{ZrO}_{2}$ (like in Figure 2 on the right), whereas the oxidized Laves phase SPPs have cracks or voids above and sometimes below. Calculations show that the volume expansion of the oxidation of $\mathrm{Zr}_{2} \mathrm{Fe}$ is more than for $\mathrm{Zr}$, which would put the precipitate in compression and the surrounding $\mathrm{ZrO}_{2}$ in tension, resulting in cracking. On the other hand, the fact that the Fe stays metallic when the Laves phase 
precipitates are oxidized means that the volume expansion here is less than that for the $\mathrm{Zr} \rightarrow \mathrm{ZrO}_{2}$ transformation, resulting in voids being created at the tops and bottoms of the oxidized SPPs. Thus, the presence of SPPs in the metal will inevitably result in cracking of the oxide scale during the corrosion process. This may contribute to the increased corrosion rates observed with alloying but also to the predictability of the corrosion rates as compared to pure zirconium [7].

\section{References:}

[1] P. Chemelle et al., Journal of Nuclear Materials 113 (1983), p. 58.

[2] X. Meng and D.O. Northwood, S, Journal of Nuclear Materials 168 (1989), p. 125.

[3] D. Pêcheur et al., Journal of Nuclear Materials 189 (1992), p. 318.

[4] D. Pêcheur, Journal of Nuclear Materials 278 (2000), p. 195.

[5] C. Proff, S. Abolhassani, and C. Lemaignan, Journal of Nuclear Materials 416 (2011), p. 125.

[6] B. de Gabory, A.T. Motta, and K. Wang, Journal of Nuclear Materials 456 (2015), p. 272.

[7] The authors acknowledge funding from AMEC Foster Wheeler and from the EPSRC (via the provision of a studentship to KJA).

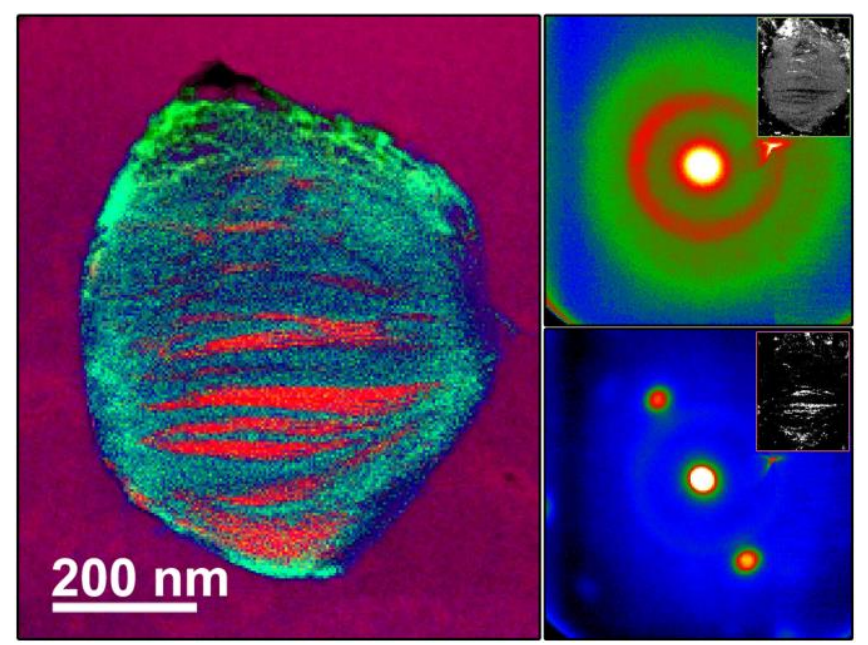

Figure 1. Chemical map of an oxidized $\mathrm{Fe}(\mathrm{Cr}, \mathrm{Fe})_{2}$ precipitate: iron (red), green (chromium) and purple (zirconium) together with diffraction patterns from the chromium-rich body (top) and the iron-rich veins (bottom).
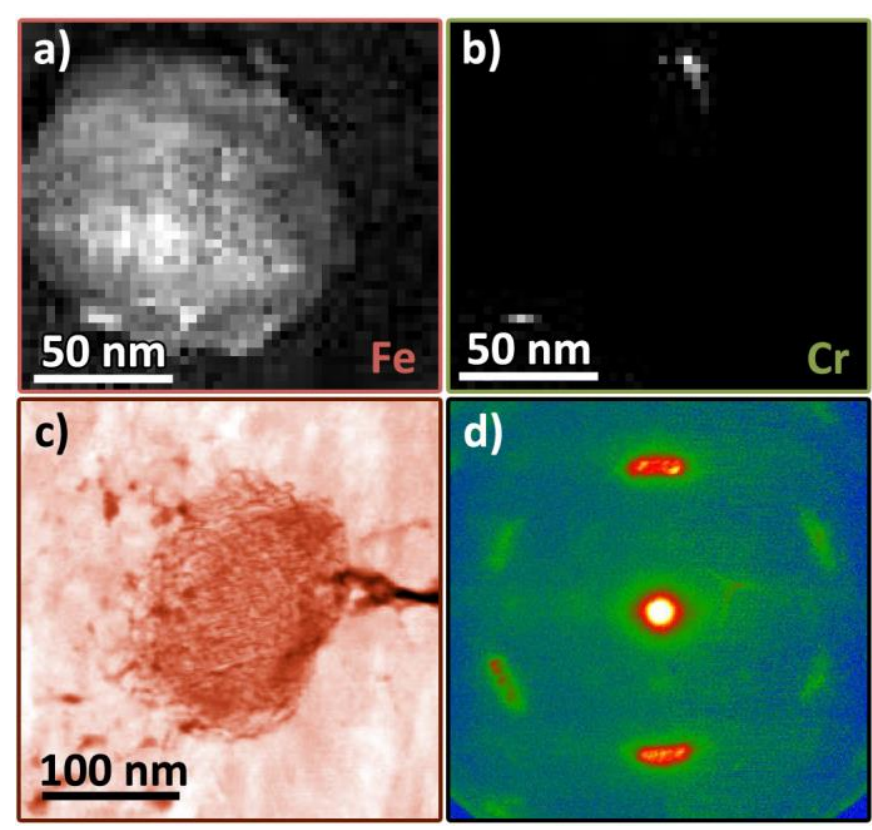

\section{d)}

Figure 2. Microscopy of oxidized $\mathrm{Zr}_{2} \mathrm{Fe}$ SPPs: a) iron map of one such SPP; b) Cr map of the same SPP showing almost no Cr, apart from at the tip; c) HAADF image of another such SPP showing the nanoscale internal structure; d) diffraction pattern from the SPP showing a strong crystallographic texture but also showing arcs at each diffraction order, demonstrating that it is made of many similarly oriented crystallites. 